\title{
FORMANTES VOCÁLICOS EN EL NGÄBERE DE COSTA RICA
}

\author{
Vowel formants in the Ngäbere of Costa Rica
}

Haakon S. Krohn ${ }^{1}$

\begin{abstract}
RESUMEN
En este artículo se presentan los resultados de un análisis acústico de la pronunciación cuidada de los ocho fonemas vocálicos orales del ngäbere de Costa Rica. Con base en las frecuencias promedios de los primeros dos formantes, se discuten las propiedades fonéticas de las vocales y sus implicaciones para la clasificación de los fonemas. Estos hallazgos se parangonan con descripciones del sistema vocálico publicadas anteriormente.

Palabras clave: Lengua ngäbere, fonética acústica, formantes vocálicos, fonología, fonemas vocálicos.
\end{abstract}

\begin{abstract}
This paper presents the results of an acoustic analysis of the eight oral vowel phonemes in the Ngäbere of Costa Rica pronounced carefully. Based on the average frequencies of the first two formants, we discuss the phonetic properties of the vowels and their implications for the classification of the phonemes. These findings are compared to earlier published descriptions of the vowel system.
\end{abstract}

Key Words: Ngäbere language, acoustic phonetics, vowel formants, phonology, vowel phonemes.

\section{Introducción}

Este trabajo consiste en un análisis acústico de los formantes de las vocales orales del ngäbere hablado en Costa Rica. Aunque el sistema vocálico de esta lengua ha sido descrito en numerosas ocasiones, nunca se han reportado las frecuencias de los formantes. Asimismo, en las descripciones publicadas anteriormente hay cierta vacilación en la clasificación de los fonemas vocálicos. Por medio de datos cuantitativos extraídos de grabaciones de habla cuidada, se pretende determinar las propiedades fonéticas de las vocales ngäberes de manera más precisa, lo cual también servirá como fundamento para una revisión de las descripciones fonológicas existentes. No se tomaron en cuenta las vocales nasales, ya que todas las reseñas anteriores concuerdan en que cada una de estas corresponde a una de las vocales orales en cuanto a todas las características excepto la nasalidad.

El análisis se realizó con base en grabaciones de la lectura de sílabas ngäberes por parte del hablante nativo Luis Quirós Palacios, residente de la comunidad de Alto Laguna en el territorio indígena de Osa, a quien le extendemos nuestros sinceros agradecimientos por su participación en este proyecto y por su hospitalidad. Las sílabas fueron seleccionadas con el

\footnotetext{
${ }^{1}$ Universidad de Costa Rica. Escuela de Filología, Lingüística y Literatura. Costa Rica.

Correo eletrónico: hkrohn@gmail.com
} 
fin de que todas las vocales se pronunciaran en entornos uniformes, con mínima influencia de sonidos contiguos.

El ngäbere, ${ }^{2}$ también conocido bajo los nombres de guaymí, ngäbe, nove, movere y varios otros, pertenece (junto con el buglé, o bocotá) al subgrupo guaimíico del grupo ístmico de la familia lingüística chibcha (Constenla Umaña, 2008, p. 127). La mayoría de los hablantes, alrededor de 150000 (Murillo, 2016, p. 9), vive en Panamá. ${ }^{3}$ En Costa Rica se registraron 9543 miembros de la etnia ngäbe/guaymí en el Censo Nacional de Población y Vivienda realizado en el 2011, de quienes un 67,7 \% reportó hablar un idioma indígena (Fuentes, 2014, pp. 332-333), lo cual daría un total de aproximadamente 6400 hablantes costarricenses. Este número difiere mucho del censo del 2000, en el que se registraron menos de 2200 hablantes en el país (Solano Salazar, 2004, pp. 363, 365). ${ }^{4}$ Los hablantes del ngäbere en Costa Rica residen principalmente en los territorios indígenas de Conteburica, Coto Brus, Abrojos-Montezuma, Altos de San Antonio y Osa, ubicados en el sur del país. Los primeros asentamientos ngäbes en Costa Rica aparecieron en los años 50 del siglo pasado como resultado de una serie de migraciones desde Panamá (Murillo Miranda, 2016, p. 10).

\section{Antecedentes}

En el presente apartado se exponen las representaciones del sistema vocálico oral del ngäbere en publicaciones anteriores. Se enfatiza en las descripciones de la variedad costarricense, pero también se incluye la de Quesada Pacheco (2008) de la variedad panameña, ya que forma parte de una gramática extensa y reciente. Cada tabla presentada contiene los símbolos fonéticos empleados por el autor original.

Entre las primeras descripciones se encuentra la de Lininger Ross (1981), cuya clasificación de los fonemas vocálicos orales del ngäbere costarricense se reproduce en la tabla $1 .^{5}$ Se observa que los divide en dos anteriores y seis posteriores; estos últimos se distinguen de acuerdo con tres niveles de altura y el redondeamiento.

\footnotetext{
${ }^{2}$ Ngäbere /yد'bere/ es una adjetivización del sustantivo ngäbe 'gente' (Quesada Pacheco, 2008, p. 15).

${ }^{3}$ De un total de 260058 personas identificadas como ngäbes en los censos de población y vivienda de Panamá de 2010 (Instituto Nacional de Estadística y Censo de Panamá, 2015, p. 40).

${ }^{4}$ El 84,5 \% de los 2563 ngäbes registrados afirmaron hablar una lengua indígena. Sin embargo, este número solo abarca a las personas residentes en territorios indígenas ngäbes. También es importante señalar que en ninguno de los dos censos citados hay un rubro propio para los buglés, quienes también habitan en los territorios ngäbes, pero hablan su propia lengua.

${ }^{5} \mathrm{La}$ información proviene de la página 103 de Lininger Ross (1981), ya que en la tabla de la página 104 solo aparecen siete de los ocho fonemas vocálicos orales mencionados anteriormente.
} 
Tabla 1

Los fonemas vocálicos orales del ngäbere

costarricense según Lininger Ross (1981)

\begin{tabular}{|c|c|c|c|}
\hline & \multirow{2}{*}{$\begin{array}{l}\text { Anteriores } \\
\text { No redond. }\end{array}$} & \multicolumn{2}{|c|}{ Posteriores } \\
\hline & & $\begin{array}{c}\text { No } \\
\text { redond. }\end{array}$ & Redond. \\
\hline Altas & $\mathrm{i}$ & $\ddot{i}$ & $\mathrm{u}$ \\
\hline Medias & $\mathrm{e}$ & $\ddot{\mathrm{e}}$ & $\mathrm{O}$ \\
\hline Bajas & & $\mathrm{a}$ & כ \\
\hline
\end{tabular}

Abarca González (1985), también con base en datos proporcionados por hablantes costarricenses, presenta la clasificación apreciada en la tabla $2 .{ }^{6}$ Las diferencias respecto a la anterior estriban en que la vocal baja no redondeada, /a/, se considera anterior en vez de posterior y en que una de las vocales posteriores no redondeadas, $/ \mathrm{x} /$, se posiciona entre media y baja.

Tabla 2

Los fonemas vocálicos orales del ngäbere

costarricense según Abarca González (1985)

\begin{tabular}{cccc}
\hline & Anteriores & \multicolumn{2}{c}{ Posteriores } \\
\hline & No redond. & No & Redond. \\
& & redond. & \\
\hline Alto & i & u & $\mathrm{u}$ \\
\hline Medias & e & \multirow{2}{*}{ r } & o \\
\hline Bajo & a & & J \\
\hline Fuente: Tomado de Abarca González (1985).
\end{tabular}

Fuente: Tomado de Abarca González (1985).

Quesada Pacheco (2008), en su gramática de la variedad de Panamá, presenta los fonemas vocálicos como se expone en la tabla 3. Esta clasificación es casi equivalente a la de Abarca González (1985), con la diferencia de que / / / se especifica como media.

Tabla 3

Los fonemas vocálicos orales del ngäbere

panameño según Quesada Pacheco (2008)

\begin{tabular}{cccc}
\hline & Anteriores & \multicolumn{2}{c}{ Posteriores } \\
\hline & No redond. & No & Redond. \\
& & redond. & \\
\hline Altas & $\mathrm{i}$ & $\mathrm{u}$ & $\mathrm{u}$ \\
\hline Medias & $\mathrm{e}$ & $\mathrm{r}$ & $\mathrm{o}$ \\
\hline Bajas & $\mathrm{a}$ & & $\mathrm{J}$ \\
\hline
\end{tabular}

Fuente: Tomado de Quesada Pacheco (2008).

\footnotetext{
${ }^{6}$ Abarca González (1985) emplea los términos bemolizada y sostenida en lugar de redondeada y no redondeada, pero los se cambiaron aquí por motivos de uniformidad.
} 
La descripción más reciente del ngäbere costarricense es la de Murillo Miranda (2016), quien realiza la clasificación apreciada en la tabla 4. Esta presenta una diferencia llamativa respecto de todas las demás: las vocales que por otros lingüistas han sido clasificadas como posteriores no redondeadas son etiquetadas como centrales por Murillo Miranda. Además, este autor afirma que la variedad costarricense se distingue de la panameña por tener una vocal posterior abierta / $\mathrm{p} /$ en vez de una posterior medio abierta $/ \mathrm{J} /$.

Tabla 4

Los fonemas vocálicos orales del ngäbere

costarricense según Murillo Miranda (2016)

\begin{tabular}{cccc}
\hline & Anteriores & Centrales & Posteriores \\
\hline & No redond. & No redond. & Redond. \\
\hline Altas & $\mathrm{i}$ & $\mathrm{u}$ & $\mathrm{u}$ \\
\hline Medias & $\mathrm{e}$ & $\mathrm{r}$ & $\mathrm{o}$ \\
\hline Bajas & $\mathrm{a}$ & & $\mathrm{D}$ \\
\hline Fuente: Tomado de Murillo Miranda (2016).
\end{tabular}

\section{Método}

\subsection{Material}

En una indagación de este tipo, es preferible que cada uno de los fonemas en cuestión se analice en los mismos entornos, dado que pueden ser influidos tanto por los sonidos que los rodeen como por factores suprasegmentales (en el caso del ngäbere, la posición con respecto al acento). Debido a que no se logró encontrar conjuntos mínimos de palabras en los que todos los fonemas vocálicos se hallaran en exactamente el mismo entorno, se decidió utilizar un corpus de sílabas sueltas, todas existentes en palabras ngäberes. Considerando la uniformidad del corpus y el estilo de lectura cuidada, las realizaciones grabadas deben de hallarse cerca de lo que podría considerarse la pronunciación prototípica de cada fonema.

En la selección de las sílabas para el corpus influyeron varios factores. En primer lugar, las sílabas del ngäbere son abiertas en la mayoría de los casos (Murillo Miranda, 2016, p. 26), por lo que todas las incluidas en el corpus son de este tipo. En segundo lugar, se optó por que el ataque silábico consistiera en una consonante oclusiva, para que el inicio de la vocal se distinguiera claramente. Se averiguó que las oclusivas /t/, /d/, /k/ y /g/ son las que pueden aparecer antes de todos los fonemas vocálicos orales. De estas cuatro, se eligió una de cada punto de articulación: /t/ (dentoalveolar) y /k/ (velar). Por consiguiente, el corpus con el que se trabajó contenía las representaciones ortográficas de las sílabas presentadas en la tabla 
5. La ortografía empleada es la que se utiliza en las clases de ngäbere en las escuelas en Costa Rica, y la misma que se ha estado empleando para escribir esta lengua en Panamá desde los años 70 (Constenla Umaña, 1996, p. 210; Lininger Ross, 1981, p. 114). En las representaciones fonológicas, incluidas en la tabla 5 solo por el motivo de ilustrar las correspondencias entre grafemas y fonemas, se usan los mismos símbolos fonéticos que en Abarca González (1985) y Quesada Pacheco (2008), puesto que se consideran en este artículo como las fuentes que mejor reflejan las realizaciones promedios, como se explicará más adelante.

Tabla 5

Silabas ngäberes incluidas en el corpus

\begin{tabular}{cc}
\hline $\begin{array}{c}\text { Repr. } \\
\text { ortográfica }\end{array}$ & Repr. fonológica \\
\hline ti & $/ \mathrm{ti} /$ \\
\hline te & $/ \mathrm{te} /$ \\
\hline $\mathrm{ta}$ & $/ \mathrm{ta} /$ \\
\hline $\mathrm{tä}$ & $/ \mathrm{t} /$ \\
\hline $\mathrm{tö}$ & $/ \mathrm{tr} /$ \\
\hline $\mathrm{tü}$ & $/ \mathrm{tu} /$ \\
\hline $\mathrm{to}$ & $/ \mathrm{to} /$ \\
\hline $\mathrm{tu}$ & $/ \mathrm{tu} /$ \\
\hline $\mathrm{ki}$ & $/ \mathrm{ki} /$ \\
\hline $\mathrm{ke}$ & $/ \mathrm{ke} /$ \\
\hline $\mathrm{ka}$ & $/ \mathrm{ka} /$ \\
\hline $\mathrm{kä}$ & $/ \mathrm{kJ} /$ \\
\hline $\mathrm{kö}$ & $/ \mathrm{kr} /$ \\
\hline $\mathrm{kü}$ & $/ \mathrm{ku} /$ \\
\hline $\mathrm{ko}$ & $/ \mathrm{ko} /$ \\
\hline $\mathrm{ku}$ & $/ \mathrm{ku} /$ \\
\hline
\end{tabular}

Fuente: Elaboración propia.

\subsection{Participante}

Como anteriormente se ha mencionado, las muestras analizadas provienen de grabaciones del señor Luis Quirós Palacios, quien reside en la comunidad de Alto Laguna en el territorio indígena de Osa, ubicado en el cantón homónimo. Alto Laguna se localiza al límite del Parque Nacional Corcovado, a unos 7 kilómetros en línea recta al suroeste del pueblo de La Palma del cantón de Golfito. De acuerdo con el Censo Nacional de Población y 
Vivienda de 2011, el $87 \%$ de los 108 habitantes indígenas del territorio de Osa declara hablar un idioma indígena (Fuentes, 2015, p. 317, 339).

Don Luis nació en el distrito de Limoncito cerca de San Vito en el cantón de Coto Brus, pero ha vivido casi toda su vida en Alto Laguna. En el momento de realizarse las grabaciones, tenía 65 años de edad. Es bilingüe en ngäbere y español, con el ngäbere como lengua materna, y maneja la ortografía práctica de esta lengua. Además, es participante activo en proyectos de revitalización lingüística y cultural en su comunidad.

\subsection{Procedimiento}

Las grabaciones se llevaron a cabo en la casa de don Luis en Alto Laguna. Las representaciones ortográficas de las sílabas del corpus se le fueron mostrando, una por una, con las indicaciones previas de que las pronunciara de manera clara y cuidada. Después de haber grabado su pronunciación de todas las dieciséis sílabas, el procedimiento se repitió, para obtener un total de cuatro muestras de cada vocal (dos de cada sílaba).

Se utilizó un micrófono digital de Radioshack con conexión USB y una respuesta de frecuencia de $70 \mathrm{~Hz}$ a $17 \mathrm{kHz}$. Durante las grabaciones también se empleó el programa Audacity (versión 2.2.1; Audacity Team, 2018), por medio del cual se guardaron los audios en formato WAV.

\subsection{Análisis}

Los archivos de audio se analizaron en el software Praat (Boersma y Weenink, 2017) para determinar las frecuencias de los dos primeros formantes (F1 y F2) de las vocales. Esta tarea se realizó manualmente, como se detalla a continuación.

De cada muestra, se visualizó el espectrograma de la grabación y se seleccionó un segmento cerca del centro de la vocal en el que los formantes estuvieran lo más estables posible. Seguidamente, se hizo uso de la función de listado de formantes ("Formant listing"), la cual extrae la frecuencia de los formantes cada 6,25 milisegundos a lo largo de la selección. De estos valores se calculó la frecuencia media de los formantes dentro del segmento seleccionado, cifras que se anotaron en una hoja de cálculo. Después del registro de las frecuencias de los dos primeros formantes de todas las muestras de las vocales, se sacó el promedio de los cuatro valores de cada formante agrupado por fonema. 


\section{Resultados}

Las frecuencias medias del F1 y el F2 de las realizaciones de cada fonema vocálico se muestran en la tabla 6. Además, se incluye el margen de error de estas estimaciones, con base en un nivel de confianza del $95 \%$. $^{7}$

Tabla 6

Frecuencias promedios del F1 y el F2 de los fonemas vocálicos orales

\begin{tabular}{ccccc}
\hline \multirow{2}{*}{ Voca } & \multicolumn{3}{c}{ F1 } & F2 \\
\cline { 2 - 5 } I & $\begin{array}{c}\text { Promedio } \\
\mathbf{( H z )}\end{array}$ & Marg. de error $(\mathbf{H z})$ & $\begin{array}{c}\text { Promedio } \\
\mathbf{( H z )}\end{array}$ & Marg. de error (Hz) \\
\hline /a/ & 760 & $\pm 34,7$ & 1432 & $\pm 49,6$ \\
\hline / / & 620 & $\pm 44,8$ & 949 & $\pm 79,8$ \\
\hline /e/ & 413 & $\pm 26,9$ & 2153 & $\pm 102,8$ \\
\hline$/ \mathrm{i} /$ & 302 & $\pm 28,9$ & 2291 & $\pm 99,4$ \\
\hline$/ \mathrm{d} /$ & 450 & $\pm 32,6$ & 735 & $\pm 107,0$ \\
\hline$/ \mathrm{y} /$ & 398 & $\pm 52,1$ & 1344 & $\pm 102,4$ \\
\hline$/ \mathrm{u} /$ & 322 & $\pm 1,2$ & 744 & $\pm 117,1$ \\
\hline$/ \mathrm{u} /$ & 280 & $\pm 80,2$ & 1511 & $\pm 108,0$ \\
\hline
\end{tabular}

Fuente: Elaboración propia.

La manera más adecuada de visualizar estos valores gráficamente es en un diagrama de dispersión, ya que las frecuencias de los formantes se relacionan con las representaciones tradicionales de altura y anterioridad/posterioridad vocálica. La frecuencia del F1 presenta una correlación inversa con la altura, mientras que la frecuencia del F2 aumenta en función de la anterioridad. No obstante, la segunda de estas dos correlaciones es menos exacta, pues el F2 también se ve afectado tanto por el redondeamiento como por la altura de la vocal (Ladefoged y Johnson, 2011, p. 196); especialmente, una vocal posterior redondeada presenta un F2 de una frecuencia considerablemente menor que una posterior no redondeada (cf. Catford, 2001, p. 154).

En la figura 1 se muestra el diagrama en el que fueron marcados los valores medios de los formantes de cada vocal, con la frecuencia del F1 en el eje vertical y la del F2 en el eje horizontal. Es necesario aclarar que los valores de las frecuencias en hercios no representan perfectamente cómo se perciben las diferencias entre las vocales, puesto que el oído humano es más sensitivo en el extremo inferior de la escala (Ladefoged, 1996, p. 78). Por esta razón, los números están convertidos a la escala Bark, ${ }^{8}$ en la que cada intervalo representa la misma

\footnotetext{
${ }^{7}$ Se utilizó el valor $t$ de 2,57, debido al bajo número de muestras.

${ }^{8}$ La escala Bark fue propuesta por Zwicker (1961). La fórmula utilizada para la elaboración del diagrama (figura 1) es la propuesta por Zwicker y Terhardt (1980): $\operatorname{Bark}=13 \arctan (0,00076 f)+3,5 \arctan (f / 7500)^{2}$.
} 
diferencia percibida. Ambos ejes están organizados de acuerdo con esta escala, por lo que los valores en hercios incluidos en el diagrama solo son aproximaciones.

Además, los ejes están invertidos para que el diagrama corresponda a las representaciones gráficas tradicionales de las vocales, con las altas arriba y las anteriores a la izquierda. El diagrama también incluye barras de error, correspondientes a los márgenes de error señalados en la tabla 6 . A este respecto, es importante tener claro que lo indicado por las barras no es el área de las posibles realizaciones de cada fonema, sino el área en la que la realización promedio del fonema se encuentra con un $95 \%$ de probabilidad.

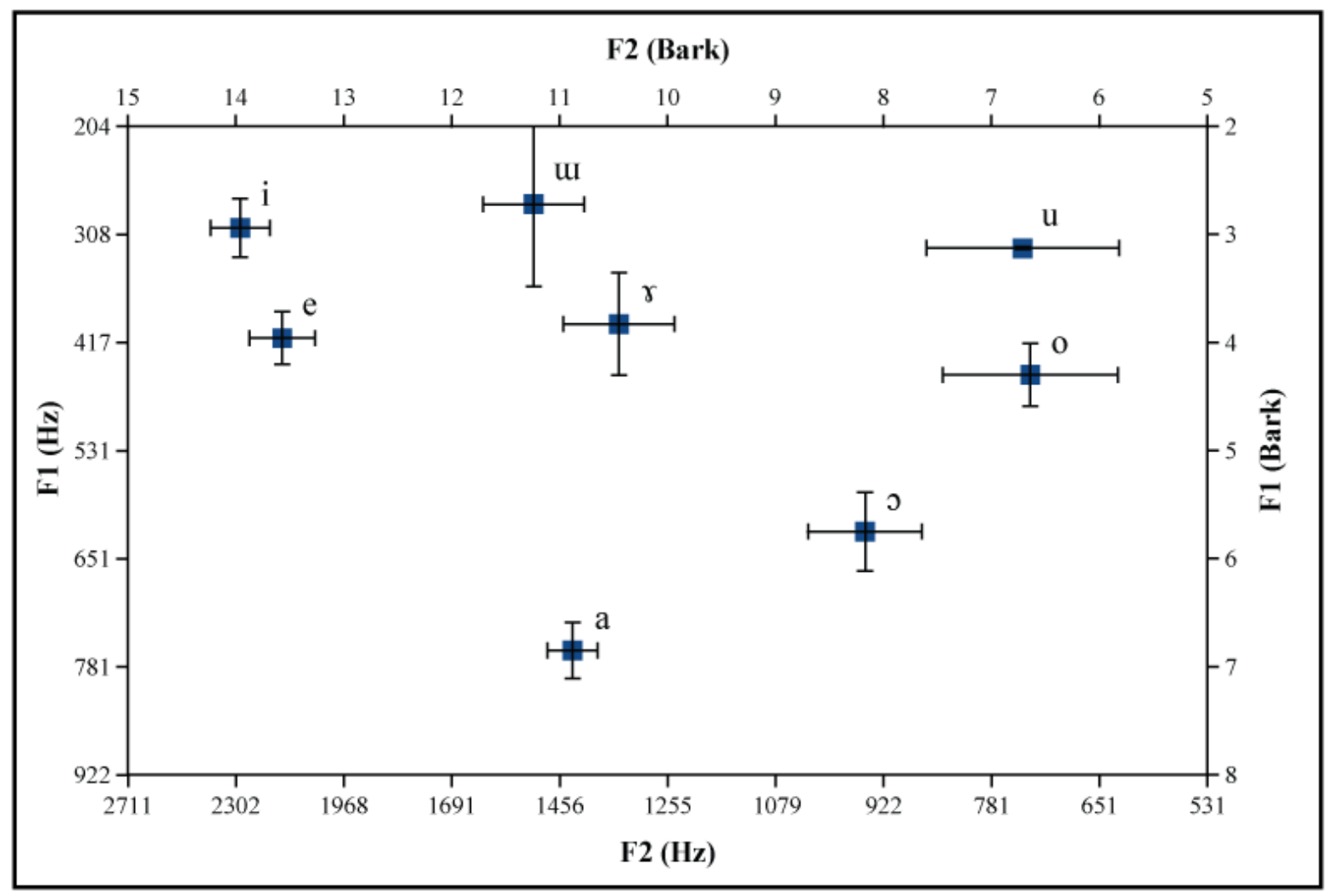

Figura 1. Diagrama con frecuencias promedios de las realizaciones de los fonemas vocálicos orales del ngäbere

\section{Discusión}

\subsection{Consideraciones fonéticas}

En este apartado se discuten las implicaciones de los resultados para la caracterización fonética de las vocales ngäberes. Nos fundamentaremos principalmente en Catford (2001), quien proporciona datos que ayudan a clasificarlas respecto a las vocales cardinales. La tabla 
7 contiene las estimaciones de dicho autor (p. 154) de las frecuencias de las vocales cardinales pronunciadas por un varón.

Tabla 7

Valores promedios propuestos por Catford para

las vocales cardinales pronunciadas por un varón

\begin{tabular}{cccccc}
\hline Vocal & $\begin{array}{c}\text { F1 } \\
\text { (Hz) }\end{array}$ & $\begin{array}{c}\text { F2 } \\
\text { (Hz) }\end{array}$ & Vocal & $\begin{array}{c}\text { F1 } \\
\text { (Hz) }\end{array}$ & $\begin{array}{c}\text { F2 } \\
\text { (Hz) }\end{array}$ \\
\hline$[\mathrm{i}]$ & 240 & 2400 & {$[\mathrm{y}]$} & 235 & 2100 \\
\hline$[\mathrm{e}]$ & 390 & 2300 & {$[\varnothing]$} & 370 & 1900 \\
\hline$[\varepsilon]$ & 610 & 1900 & {$[\propto]$} & 585 & 1710 \\
\hline$[\mathrm{a}]$ & 850 & 1610 & {$[\mathrm{\leftarrow}]$} & 820 & 1530 \\
\hline$[\mathrm{a}]$ & 750 & 940 & {$[\mathrm{p}]$} & 700 & 760 \\
\hline$[\Lambda]$ & 600 & 1170 & {$[\mathrm{\jmath}]$} & 500 & 700 \\
\hline$[\mathrm{x}]$ & 460 & 1310 & {$[\mathrm{o}]$} & 360 & 640 \\
\hline$[\mathrm{u}]$ & 300 & 1390 & {$[\mathrm{u}]$} & 250 & 595 \\
\hline
\end{tabular}

Fuente: Tomado de Catford (2001), p. 154.

En el análisis del ngäbere de la presente investigación, las vocales /i/, /w/ y /u/ destacan, como era de esperar, como las tres más altas (aunque el margen de error de /u/ parcialmente coincide con el de $/ \mathrm{\gamma} /$ ), y se hallan aproximadamente a la misma altura. De estas, /i/ y /u/ son claramente anteriores y posteriores, respectivamente, por lo que no se alejan mucho de las vocales cardinales [i] y [u]. En cuanto a /u/, la clasificación es menos evidente, dado que sus realizaciones se encuentran en una posición intermedia, incluso más cerca de /i/ que de /u/ en la escala Bark. Sin embargo, la baja frecuencia del F2 de las realizaciones de /u/ se debe, en gran medida, al redondeamiento, de ahí que la visualización en la figura 1 sea parcialmente engañosa. Catford (2001, p. 155), para las vocales altas redondeadas, define el límite entre posteriores y centrales a alrededor de $1050 \mathrm{~Hz}$, mientras que el de las correspondientes no redondeadas lo traza a casi $1500 \mathrm{~Hz}$. Por lo tanto, la realización promedio de /w/ se encuentra justamente alrededor de este límite, es decir, en un punto intermedio entre las vocales cardinales [u] y [i]].

Seguidamente, se observa que la realización media de /e/ se ubica cerca de la vocal cardinal [e], mientras que la de /o/ se halla entre [o] y [0], por lo que esta se asemeja más a una vocal media. El fonema $/ \mathrm{\gamma} /$, por su lado, parece central en la figura 1, pero debido a su falta de redondeamiento se clasificaría definitivamente como posterior de acuerdo con Catford (2001); se encuentra cerca de la vocal cardinal [x], pero es un poco más alta. 
La pronunciación del fonema / $/$ / no se asocia directamente con ninguna vocal cardinal. En lo que concierne a su altura, se halla entre [0] y [p], lo cual explica las discrepancias entre la clasificación de Murillo Miranda (2016) y las demás. Asimismo, es más anterior que las vocales cardinales posteriores redondeadas, pero no tanto como para considerarse central.

Por último, la realización media de /a/ es considerablemente más baja que la de / $/$ /, tanto que se podría afirmar que se encuentra en un nivel de altura diferente. Además, debido a la alta frecuencia de su F2, puede clasificarse como anterior de acuerdo con lo expuesto por Catford (2001, p. 155), aunque no es tan anterior como la vocal cardinal [a]. Por lo tanto, se describiría mejor como baja anterior centralizada y su pronunciación promedio podría representarse como [ä].

\subsection{Consideraciones fonológicas}

Los hechos tratados en el apartado 5.1 muestran que las propiedades fonéticas de cada vocal son variables y que difícilmente pueden describirse por medio de categorías discretas. En las descripciones fonológicas, sin embargo, es de mayor relevancia clasificar los fonemas de tal manera, con el fin de señalar las oposiciones funcionales.

En cuanto a /i/, /e/, /u/ y /o/, es evidente que se trata de dos fonemas anteriores y dos posteriores, de dos niveles de altura diferentes. Nos enfocaremos, por lo tanto, en los fonemas que en publicaciones anteriores han sido clasificados inconsistentemente. La primera cuestión es si / w/ y / $/$ / deben considerarse centrales o posteriores. Como señala Odden, los fonólogos distinguen principalmente entre vocales anteriores y posteriores: "Phonetic central vowels are usually treated as phonological back vowels, since typically central vowels are unrounded and back vowels are rounded" (2013, p. 50). Teniendo esto en cuenta, está claro que no es necesario postular vocales centrales fonológicas en el ngäbere. Tanto / $\mathrm{w} /$ como / $/ \mathrm{\gamma}$, que fonéticamente se acercan a una posición central, se distinguen de /u/ y /o/ principalmente por la característica [-redondeada], mientras que la centralidad es un rasgo secundario y fonológicamente irrelevante.

También está el interrogatorio de cuántas alturas fonológicas se distinguen en la variedad lingüística estudiada. Aunque fonéticamente destacan al menos cuatro niveles, solo hacen falta tres para definir las oposiciones fonológicas, como ha sido reflejado en todas las publicaciones anteriores. Los fonemas $/ \mathrm{a} / \mathrm{y} / \mathrm{J} / \mathrm{se}$ diferencian entre sí principalmente por el redondeamiento (al igual que las oposiciones $/ \mathrm{w} / \neq / \mathrm{u} / \mathrm{y} / \mathrm{\gamma} / \neq / \mathrm{o} /$ ), aunque a nivel fonético también son notablemente divergentes en lo que concierne tanto a la altura como al grado de 
anterioridad. De hecho, también resulta irrelevante especificar fonológicamente si /a/ es anterior o posterior, puesto que este fonema se distingue de todos los demás por ser el único que presenta los rasgos [+baja, -redondeada].

\section{Conclusiones}

En suma, la representación de Quesada Pacheco (2008) del sistema vocálico de la variedad panameña, reproducida en la tabla 3, resulta perfectamente adecuada también para la costarricense. Los rasgos [alta], [baja], [anterior] y [redondeada] son los que se necesitan para describir todas las distinciones fonológicas entre las vocales orales, y el uso del símbolo / $/$ /, que normalmente denota una vocal semibaja, para representar una vocal fonológicamente baja redondeada refleja el hecho de que /a/ y / $/$ / fonéticamente presentan alturas diferentes. Esto también implica que el análisis realizado aquí no reveló diferencias sustanciales entre las variedades del ngäbere habladas en Costa Rica y Panamá en lo que respecta al sistema vocálico. No obstante, es importante recordar que el habla analizada corresponde a la primera generación de ngäbes nacidos en Costa Rica, además de que hacen falta datos cuantitativos del ngäbere panameño para realizar una comparación válida.

La limitación principal del presente trabajo es el hecho de que solo se estudiaron pronunciaciones muy cuidadas de las vocales en entornos uniformes, además de que la información proviene de un solo hablante. El siguiente paso en la caracterización de las vocales del ngäbere costarricense sería un análisis de los formantes vocálicos en habla más espontánea, para dar cuenta de las áreas de dispersión de las realizaciones de cada fonema. También deberá investigarse si las características fonéticas de las vocales nasales efectivamente corresponden a las de las orales. 


\section{Referencias bibliográficas}

Abarca González, R. (1985). Análisis fonológico de guaymí movere. Estudios de Lingüística Chibcha, IV, 7-46.

Audacity Team. (2018). Audacity: Free Audio Editor and Recorder (versión 2.2.1) [software]. Recuperado de: http://www.audacityteam.org.

Boersma, P. y Weenink, D. (2017). Praat: Doing Phonetics by Computer (version 6.0.30) [software]. Ámsterdam: Department of Language and Literature, University of Amsterdam. Recuperado de: http://www.praat.com.

Catford, J. C. (2001). A practical introduction to phonetics (2. ${ }^{a}$ edición). Oxford: Oxford University Press.

Constenla Umaña, A. (1996). Poesía tradicional indígena costarricense. San José: Editorial de la Universidad de Costa Rica.

Constenla Umaña, A. (2008). Estado actual de la subclasificación de las lenguas chibchenses y de la reconstrucción fonológica y gramatical del protochibchense. Estudios de Lingüistica Chibcha, XXVII, 117-135.

Fuentes, E. (2014). Características demográficas y socioeconómicas de las poblaciones indígenas de Costa Rica (Censo 2011). En Instituto Nacional de Estadística y Censos (Ed.), Costa Rica a la luz del Censo 2011 (pp. 309-343). San José: INEC.

Instituto Nacional de Estadística y Censo de Panamá. (2015). Diagnóstico de la Población Indígena en Panamá [PDF]. Consultado el 30 de mayo de 2018 en https://www.contraloria.gob.pa/inec/archivos/P6571INDIGENA_FINAL_FINAL.pdf

Ladefoged, P. (1996). Elements of acoustic phonetics (2. ${ }^{a}$ edición). Chicago/Londres: The University of Chicago Press.

Ladefoged, P. y Johnson, K. (2011). A course in phonetics (6. ${ }^{\mathrm{a}}$ edición). Boston: Wadsworth.

Lininger Ross, B. (1981). Estudios sobre el guaymí ngäbere: fonología, alfabeto y diccionario provisional. Revista de Filología y Lingüistica de la Universidad de Costa Rica, 7 (12), 101-115.

Murillo Miranda, J. M. (2016). Gramática de la lengua guaymí. Dialecto de Costa Rica: una descripción urgente. Editorial Académica Española.

Odden, D. (2013). Introducing phonology (2. edición). Cambridge: Cambridge University Press. 
Quesada Pacheco, M. A. (2008). Gramática de la lengua guaymí (ngäbe). Múnich: Lincom Europa.

Solano Salazar, Elizabeth. (2004). La población indígena en Costa Rica según el Censo 2000. En Rosero Bixby, L. (Ed.), Costa Rica a la luz del Censo del 2000 (pp. 341-373). San José: Centro Centroamericano de Población de la Universidad de Costa Rica.

Zwicker, E. (1961). Subdivision of the audible frequency range into critical bands (Frequenzgruppen). The Journal of the Acoustic Society of America, 33 (2), 248-248.

Zwicker, E. y Terhardt, E. (1980). Analytical expressions for critical-band rate and critical bandwidth as a function of frequency. The Journal of the Acoustic Society of America, $68,1523-1524$.

\section{(). $(\Theta \Theta$}

Esta obra está bajo una licencia de Creative Commons Reconocimiento-NoComercialSinObraDerivada 4.0 Internacional 